\title{
INTENSITY DISTRIBUTION IN THE LINE EMISSION SPECTRUM OF CESIUM
}

\author{
By Fred L. Mohler
}

\section{ABSTRACT}

The intensity in a series of lines is $J_{n}=h \nu N_{n} A_{n}$, where $N_{n}$ is the number of atoms per cubic centimeter in the $n$th state, and $A_{n}$ is the rate of radiation per atom. $A_{n}$ is proportional to $n^{-3}$ for large values of $n$, while for a limited range of conditions $N_{n}$ is known to have a temperature distribution.

Line intensities were measured by direct comparison with spectra of a strip lamp. Plots of $\log J / \nu^{4}$ versus $\log n$ give parallel curves for the $\mathrm{S}, \mathrm{D}$, and $\mathrm{F}$ series, and the form of the curve is nearly independent of current and pressure for pressures above $70 \mu$. Measurements of the D series corrected for self-absorption give plots of $\log J n^{3}$ versus term energy, which are straight lines with slopes consistent with measured values of reversal temperature for the $\mathrm{P}$ series. Measurements of intensity of two D series lines over a wide range of conditions show that the intensities are proportional to the continuous spectrum intensity at the higher pressures.

For pressures above $70 \mu$ the excited states have a temperature distribution from $n=4$ to 14 . For the $\mathrm{D}$ series $A_{n}=\frac{6.4 \times 10^{8}}{n^{3}} \frac{\lambda_{n}}{5635}$. The values of $A / \nu^{3}$ are about twice the theoretical hydrogen values. The values for the $\mathrm{F}$ series are also about twice the hydrogen values, while for the $\mathrm{S}$ series they are 16 times the hydrogen values.

There is evidently an equilibrium between the number of ions and the number of excited atoms at high pressure, and in the same range of discharge conditions the temperature distribution of excited states is found.

\section{CONTENTS}

I. Introduction Page

II. Experimental results

1. Relative intensities in the $\mathrm{S}, \mathrm{D}$, and $\mathrm{F}$ series

2. Intensity of the $\mathrm{D}$ series for a columnar discharge

3. Intensity variation with current and pressure

III. Discussion

1. Population of excited states

2. Transition probabilities

3. Line spectra and continuous spectra

\section{INTRODUCTION}

This is one of a series of papers ${ }^{1}$ on the electrical discharge in cesium vapor. Published papers include data on the intensity variation of the first doublet of the principal series, on the intensity of the continuous recombination spectrum, and on the number of excited atoms in the states $6^{2} \mathrm{P}$ to $11^{2} \mathrm{P}$.

1 F. L. Mohler, Power input and dissipation in the positive column of a caesium discharge, BS J. Research 9, 35 (1932) RP455; Collisions of the first and second kind in the positive column of a caesium discharge, BS J, Research 9, 93 (1932) RP485; Recombination radiation in the cesium positive column, BS J. Research 10, 771 (1933) RP565; Reversal temperature and population of excited states in the cesium discharge, J. Research NBS 16, 227 (1936) R P869. 
This is a study of the intensity distribution in the subordinate and fundamental series. There are numerous papers on intensity distribution in line emission spectra but, in general, there are unknown factors involved which preclude a direct comparison of experiment and theory. Spectrophotometric studies ${ }^{2}$ of flames and carbon arcs containing alkali salts have, however, given some information on transition probabilities in the alkali spectra.

The number of quanta emitted per cubic centimeter per second in a transition $n l j$ to $n^{\prime} l^{\prime} j^{\prime}$ is

$$
J / h_{\nu}=N_{n l j} A_{n l j}^{n^{\prime} l^{\prime} j^{\prime}}
$$

where $J$ is the radiation in ergs, $N$ is the number of atoms per cubic centimeter in the initial state, and $A_{n}$ is the transition probability or rate of radiation per atom. Two extreme cases are of interest. If the pressure and current are so low that every atom radiates before it interacts with other atoms or electrons $N$ is proportional to $1 / A_{n}$ and $J$ is a measure of the rate of production of excited states. In the other extreme, where interactions are frequent in comparison to $1 / A_{n}$, there will be a random distribution of population between the different states $N_{n l j}$, and the population will cease to depend on $A_{n}$. This random distribution is probably established at pressures above a few microns.

For the case of thermal equilibrium at a temperature $T$,

$$
N_{n l j}=N_{a} \frac{2_{j}+1}{2 j_{a}+1} e^{-E_{n} / k T},
$$

where $N_{a}$ is the number of atoms in the normal state, $E_{n}$ the energy of the excited state, and $2 j+1$ and $2 j_{a}+1$ the weights of the two states. In another paper, ${ }^{3}$ it is shown that for a limited range of conditions the principal series lines have a nearly constant reversal temperature beyond the third line. If the reversal temperature is constant $N_{n l}$ of equation 1 can be expressed by equation 2 with $T$ equal to the reversal temperature.

Equation 1 is only applicable where self-absorption is negligible. For this reason it is not feasible to measure $J$ for the principal series and it is necessary to use the subordinate and fundamental series. For these series there are no theoretical values for the transition probabilities. $A_{n}$ is, in general, a complicated function of the quantum numbers, but for large values of $n$ it becomes proportional to $1 / n^{3}$. In this work the effective quantum number (the square root of the Rydberg denominator) will be used for $n$. This has the advantage that terms of equal $n$ values have equal energies. Furthermore, it is probable that in a comparison of transition probabilities with hydrogen the effective quantum number and not the total quantum number should be used. For the first terms of the cesium series the numbers are approximately 1.87 for $6 \mathrm{~S} ; 2.36$ and 2.33 for $6 \mathrm{P}$; 2.54 for $5 \mathrm{D}$ and 3.97 for $4 \mathrm{~F}$.

${ }_{2}^{2}$ Ornstein and Key, Physica 1, 945 (1934); Van der Held and Heierman, Physica 3, 31 (1936).

3 See footnote 1. 


\section{EXPERIMENTAL RESULTS}

\section{RELATIVE INTENSITIES IN THE S, D, AND F SERIES}

Before the systematic study of the columnar discharge was started, the author made a series of measurements of intensity of nearly all of the emission lines in the visible region, using a discharge between a hot-wire cathode and a surrounding cylindrical anode. The discharge was viewed parallel to the axis of the cylinder along a line about a centimeter from the cathode. The spectrum was photographed with a 3-prism Steinheil spectrograph. The spectrum of a calibrated strip lamp was superposed on that of the discharge and intensities measured by photographic densitometry between nearly equal densities. The line intensity for equal densities is equal to $J(\lambda) \delta \lambda$, where $J(\lambda)$ is given by Wien's law and $\delta \lambda$ is the slit width expressed in wavelength units.

At relatively high pressures there was no perceptible change in intensity distribution with change of current and pressure. Three sets of measurements with a current of about 0.5 ampere and pressures of 130,290 , and 500 microns of mercury gave intensities for 32 lines, which remained quite accurately proportional, and two arbitrary constants reduced the three sets of values to equality within experimental uncertainty. The mean values are plotted in figure 1. The logarithmic scale is used for convenience. $J / \nu^{4}$ is proportional to the matrix element, squared, times the population of the upper state. The abscissa is the $\log$ of the effective quantum number. The curves plotted are the red components of the $\mathrm{F}$ series, both components of the $\mathrm{D}$ series, and the red components of the $\mathrm{S}$ series. There is some self-absorption of the stronger lines, and the form of the curves is slightly distorted from this effect.

\section{INTENSITY OF THE D SERIES FOR A COLUMNAR DISCHARGE}

For a comparison with other experiments on the cesium discharge, intensity measurements have been repeated, using a columnar discharge in a tube $1.8 \mathrm{~cm}$ in diameter. The discharge was viewed diametrically across the tube. The intensity measurements were based on a series of exposures to a strip lamp on each plate. Selfabsorption was measured by means of a discharge tube with two anodes in parallel arms. The line of sight passed diametrically through the two arms. An exposure with both discharges running was compared with a double exposure to the two discharges separately. Except for a small difference due to reciprocity failure of the photographic plate, the higher series lines are equally dense in the two cases, while the strongest lines are weaker on the exposure with both discharges running. The correction to be applied to a single discharge tube is taken to be half the measured difference between two tubes running simultaneously and separately. Table 1 gives corrections for a few cases. The values for $5635 \mathrm{~A}$ are based on another experiment sighting across a tube and along the axis of a tube $19 \mathrm{~cm}$ long. 
TABLE 1.-Correction to be added to $\log J$ for self-absorption

\begin{tabular}{|c|c|c|c|}
\hline $\begin{array}{l}\text { 6P } \mathrm{P}_{1 / 2} 7 \mathrm{D} \text { 6724 A } \mathrm{A} \\
6 \mathrm{P}_{1}{ }_{2}-9 \mathrm{D} 5664 \mathrm{~A} \\
6 \mathrm{P}_{3 / 2}-10 \mathrm{D} 5635 \mathrm{~A}\end{array}$ & $\begin{array}{r}150 \mu, 2 \mathrm{amp} \\
0.105 \\
.040 \\
.013\end{array}$ & $\begin{array}{r}150 \mu, 0.5 \mathrm{amp} \\
0.071 \\
.018 \\
.006\end{array}$ & $\begin{array}{r}33 \mu, 2 \mathrm{amp} \\
0.08 \\
.018 \\
.006\end{array}$ \\
\hline
\end{tabular}

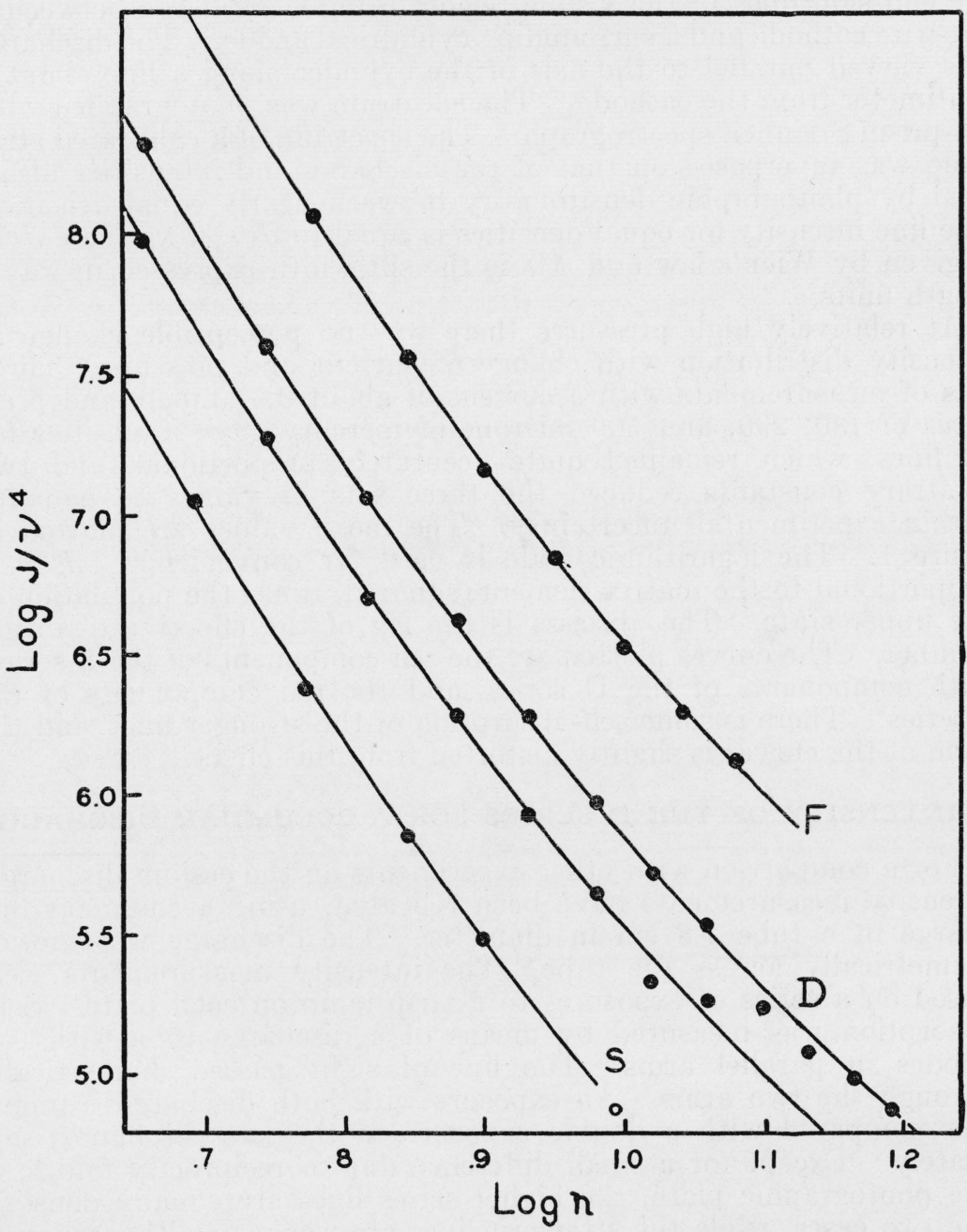

Figure 1.-Intensity distribution in the $\mathrm{S}, \mathrm{D}$, and $\mathrm{F}$ series for a high-pressure discharge.

The red components of the $\mathrm{F}$ and $\mathrm{S}$ series, and both components of the $\mathrm{D}$ series, are plotted. $J / \nu^{4}$ is in arbitrary units, and $n$ is effective quantum number.

There is an appreciable background of continuous spectrum near the limit, and correction has been applied to the last three lines for this.

Most of the measurements have been limited to seven lines of the diffuse series. Table 2 lists the lines and the values of $\log J$ and corrections for a typical series of measurements. The first two lines 
are violet components of the series, and 0.30 has been added to $\log \mathrm{J}$ to give the value of the red component.

TABLE 2.-Measurements and computations for a 1-ampere discharge at a pressure of $150 \mu$

\begin{tabular}{|c|c|c|c|c|c|c|c|}
\hline Transition & $\begin{array}{l}\text { Wave } \\
\text { length }\end{array}$ & $n$ & $\begin{array}{l}\text { Meas- } \\
\text { ured } \\
\log J\end{array}$ & $\begin{array}{l}\text { Correc- } \\
\text { tions }\end{array}$ & $\begin{array}{c}\log J \\
6 \mathrm{P}_{3 / 2}-n \mathrm{D}\end{array}$ & $\begin{array}{l}\text { Log } \\
n^{3} J\end{array}$ & $\begin{array}{l}{ }^{1} \mathrm{E}_{n} \\
\text { Electron } \\
\text { volt }\end{array}$ \\
\hline 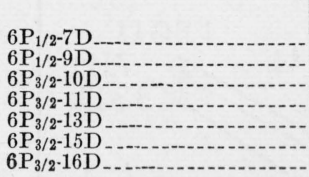 & $\begin{array}{l}\text { A } \\
6724 \\
5664 \\
5635 \\
5503 \\
5351 \\
5267 \\
5240\end{array}$ & $\begin{array}{r}4.52 \\
6.52 \\
7.52 \\
8.52 \\
10.52 \\
12.52 \\
13.52\end{array}$ & $\begin{array}{l}4.62 \\
3.44 \\
3.40 \\
3.15 \\
2.76 \\
2.47 \\
2.39\end{array}$ & $\begin{array}{r}0.30+.09 \\
.30+.03 \\
+.01 \\
-.01 \\
-.02 \\
-.06\end{array}$ & $\begin{array}{l}\text { 5. } 01 \\
\text { 3. } 77 \\
\text { 3. } 41 \\
\text { 3. } 15 \\
\text { 2. } 75 \\
\text { 2. } 45 \\
2.33\end{array}$ & $\begin{array}{l}\text { 6. } 98 \\
6.22 \\
6.04 \\
\text { 5. } 95 \\
\text { 5. } 81 \\
\text { 5. } 74 \\
\text { 5. } 73\end{array}$ & $\begin{array}{r}0.668 \\
.318 \\
.239 \\
.186 \\
.122 \\
.087 \\
.075\end{array}$ \\
\hline
\end{tabular}

${ }^{1} E_{n}$ is the negative energy of the initial state measured from the ionization potential.

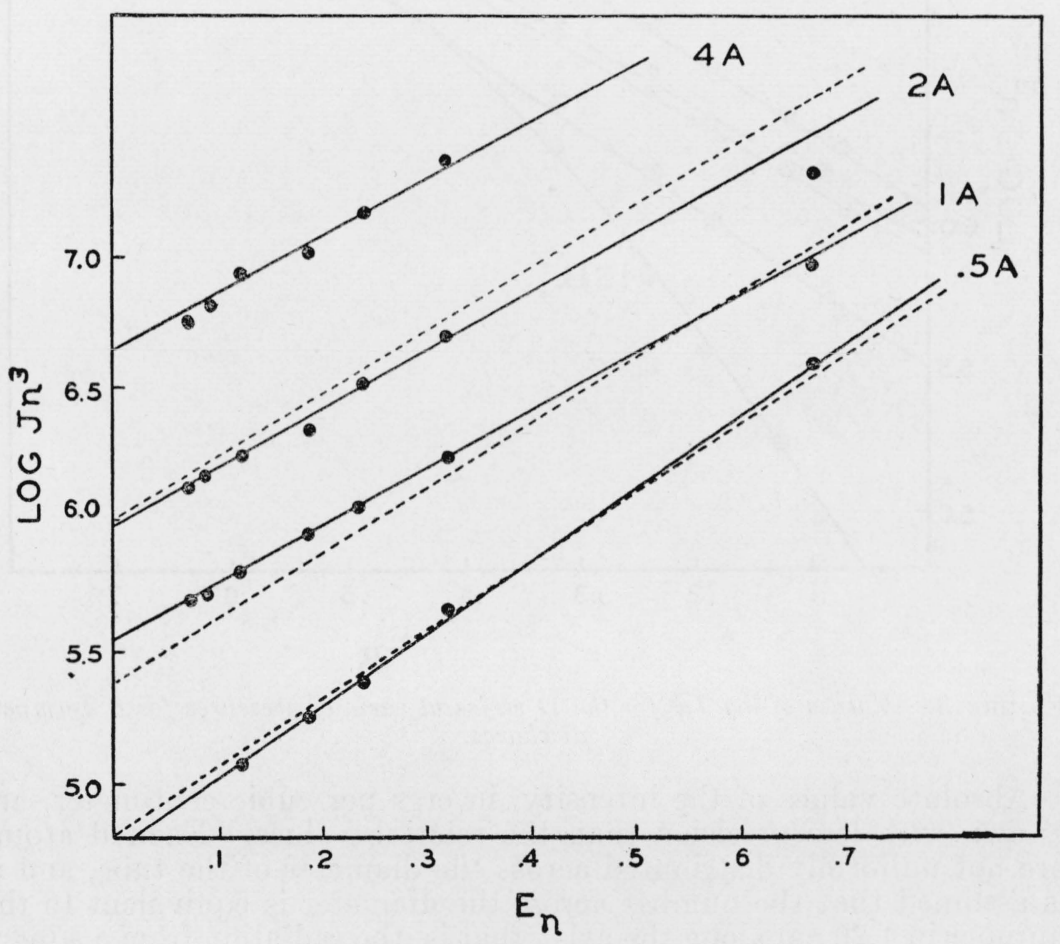

FIgURE 2.-Values of $\log J n^{3}$ for the D series for various currents at a pressure of $150 \mu$.

$E_{n}$ is the negative energy of the upper state in electron volts. Broken lines are computed from the reversal temperatures of the $\mathrm{P}$ series.

It follows from considerations given in the introduction that in each series $J n^{3}$ is proportional to the population of the states, and, if the distribution of excited states approximates a thermal distribution, a natural choice of coordinates is $\log J n^{3}$ versus the energy of the state. Figure 2 shows the results for four different currents at a pressure of 
150 microns of mercury. Figure 3 shows results at a number of pressures for a 2-ampere discharge. In Figure 2 there are included. as broken lines, the values $\log N_{n}$ computed from the observed reversal temperatures of the higher principal series lines. ${ }^{4}$ A single arbitrary constant is added to fit the three broken lines to the scale of $\log J n^{3}$.

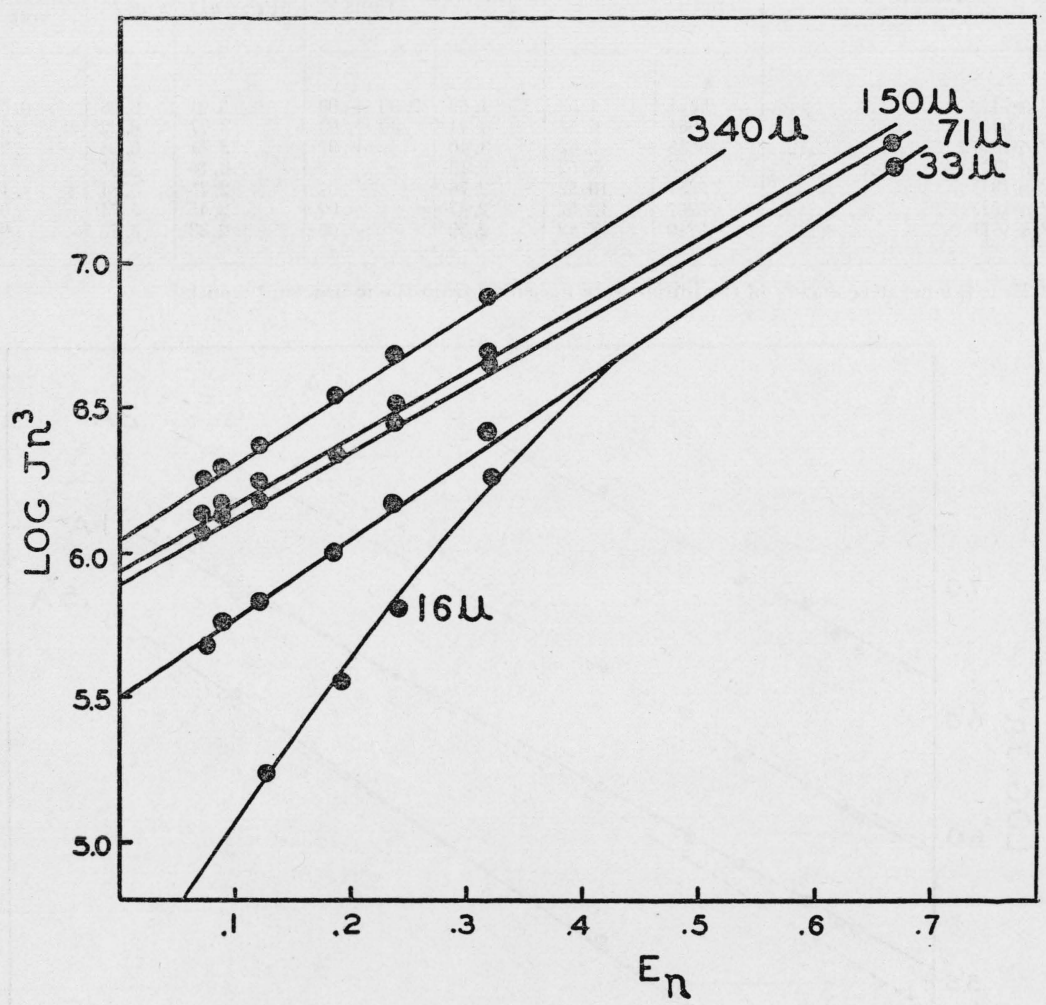

Figure 3.-Values of $\log J n^{3}$ for the $\mathrm{D}$ series at various pressures for a 2-ampere discharge.

Absolute values of the intensity, in ergs per cubic centimeter, are known with less precision than the relative values. Excited atoms are not uniformly distributed across the diameter of the tube, and it is assumed that the number across the diameter is equivalent to the number in $1.26 \mathrm{~cm}$ along the axis; that is, the radiation from a square centimeter comes from $1.26 \mathrm{~cm} .^{3}$ The plotted and tabulated values are on an arbitrary scale and 4.26 must be subtracted to obtain values of $\log J$ in terms of ergs per cubic centimeter per second.

\section{INTENSITY VARIATION WITH CURRENT AND PRESSURE}

Measurements of the intensity of two superposed diffuse series lines were made over a very wide range of current and pressure. A columnar discharge through a tube about $20 \mathrm{~cm}$ long was viewed end on by a low-dispersion spectroscope and intensity measured by

4 See footnote 1 . 
visual matching of the image of a strip lamp superposed on the image of the discharge. The settings were made on the unresolved lines $6 \mathrm{P}_{1 / 2}-10 \mathrm{D}$ at $5466 \mathrm{~A}$ and $6 \mathrm{P}_{3 / 2}-11 \mathrm{D}$ at $5503 \mathrm{~A}$. The very low dispersion permitted measurements at low intensity. Figure 4 gives results ${ }^{5}$ plotted in terms of $\log J$, in arbitrary units, versus the log of the pressure, in microns, for currents ranging from 4 to 0.3 ampere. At higher pressures the intensity of the continuous spectrum beyond the $6 \mathrm{P}$ limit is proportional to the line intensity. Broken lines show values of $\log J$ for the continuous spectrum at lower pressures. A constant has been added to $\log J$ for the continuous spectrum to make it coincide with the line-intensity values. Other lines of the diffuse series change in the same general way with pressure. For lines of higher quantum number the intensity begins to rise with pressure at a lower pressure. The fundamental series is very faint at low pressure.

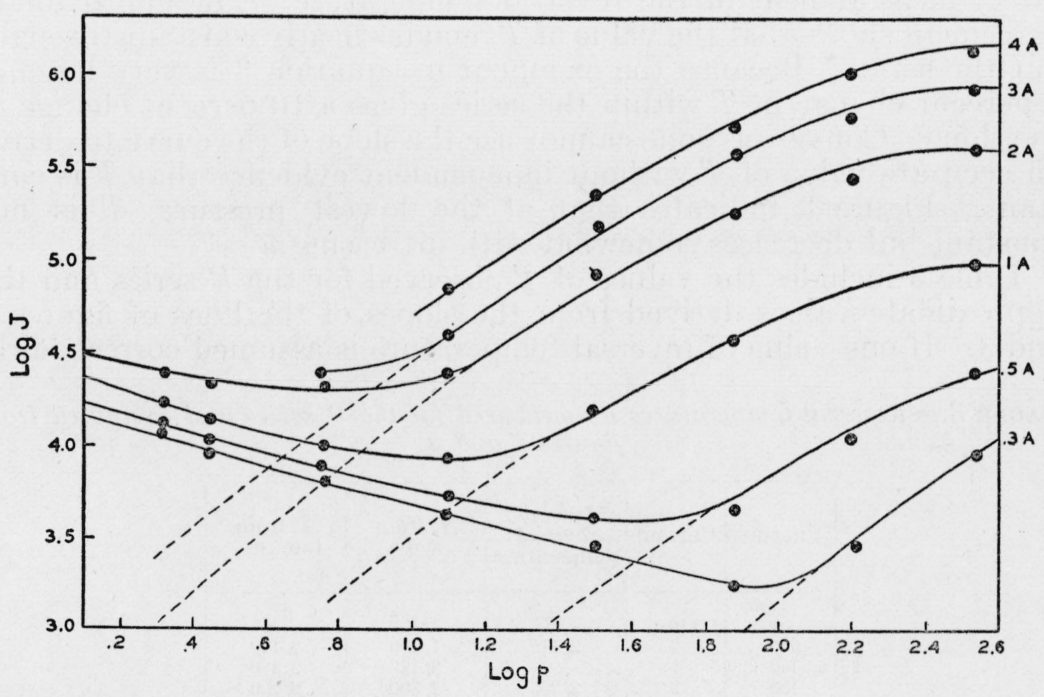

Figure 4.- - Log $J$ for diffuse series lines 5466 A and 5508 A versus log of the pressure in microns.

Broken lines give the relative intensity of the continuous spectrum below the range where it is proportional to the line spectrum.

\section{DISCUSSION}

\section{POPULATION OF EXCITED STATES}

The purpose of this study was to obtain information concerning both transition probabilities and the population of excited states, but the reasoning is complicated by the limitations of experimental and theoretical data. An inspection of theoretical hydrogen values ${ }^{6}$ of transition probabilities, matrix elements squared, etc., indicates that $A_{n} h \nu n^{3}$ remains constant even for small values of $n$, and it is assumed that it is constant for the diffuse series of cesium from $6 \mathrm{P}-7 \mathrm{D}$

\footnotetext{
s F. L. Mohler, BS J. Research 10, 776 (1931) RP565, gives the same data plotted as a function of the electron concentration.

B Bethe, Handbuchder Physik 24,442.
} 
to the limit. From equations 1 and 2 and this relation, it follows that

$$
J n^{3}=k^{\prime} N_{a} \frac{g_{n}}{g_{a}} e^{-E_{n} / k T},
$$

where $k^{\prime}$ is the constant value of $A h \nu n^{3}$, and $g$ 's are the weights of the states. For the intensity of both components of the diffuse doublets,

$$
\log J n^{3}=\log 5 k^{\prime}+\log N_{a}-5.04 \times 10^{3} E_{n} / T,
$$

where $E_{n}$ is the energy of the $n$th state in electron volts. $T$ is the reversal temperature of the $n$th line, and, if it is constant, the plot of $\log J n^{3}$ versus $E$ will give a straight line with a slope proportional to $1 / T$. Figure 2 shows a satisfactory agreement between the straight lines derived from intensity measurements and the lines based on direct measurement of the reversal temperature. The approximate agreement shows that the value of $T$ remains nearly constant throughout the series. Because the exponent in equation 3 is very large, a 1 percent change in $T$ within the series gives a 10 percent change in the slope. Conversely, one cannot use the slope of the curve to derive an accurate value of $T$ without independent evidence that $T$ is constant. Figure 3 indicates that at the lowest pressure, $T$ is not constant but decreases somewhat with increasing $n$.

Table 3 includes the values of $T$ observed for the $P$ series and the approximate values derived from the slopes of the lines of figures 2 and 3. If one value of reversal temperature is assumed correct fairly

TABLE 3.-Reversal temperatures as measured for the $\mathrm{P}$ series and computed from

\begin{tabular}{|c|c|c|c|c|}
\hline Pressure & Current & $\begin{array}{l}\text { Measured } \\
\text { reversal } \\
\text { temperature }\end{array}$ & $\begin{array}{l}T, \text { from } \\
\text { slope }\end{array}$ & $\begin{array}{c}T, \text { from } \\
\text { intensity }\end{array}$ \\
\hline $\begin{array}{r}\mu \\
340 \\
150 \\
150 \\
150 \\
150 \\
71 \\
33 \\
16\end{array}$ & $\begin{array}{c}\operatorname{amp} \\
2 \\
4 \\
2 \\
1 \\
1 \\
0.5 \\
2 \\
2 \\
2\end{array}$ & $\begin{array}{c}{ }^{\circ} \mathrm{K} \\
2,115 \\
2,205 \\
2,030 \\
1,938 \\
2,270\end{array}$ & $\begin{array}{c}{ }^{\circ} \mathrm{K} \\
1,980 \\
2,380 \\
2,300 \\
2,310 \\
1,840 \\
2,240 \\
1,860\end{array}$ & $\begin{array}{c}{ }^{\circ} \mathrm{K} \\
2,140 \\
2,380 \\
2,200 \\
2,080 \\
1,938 \\
2,260 \\
2,290 \\
2,210\end{array}$ \\
\hline
\end{tabular}
figures 2 and 3

accurate values of the relative temperatures can be computed from the difference in $\log J n^{3}$ at one wave length. From equation 4,

$$
5.04 \times 10^{3} E_{n}\left(\frac{1}{T_{2}}-\frac{1}{T_{1}}\right)=\log J_{1} n^{3}-\log J_{2} n^{3}+\log N_{a, 2}-\log N_{a, 1} \text {, }
$$

where subscripts 1 and 2 refer to two conditions of current and pressure. Table 3 gives, in the last column, values of $T$ based on the intensity of $5635 \mathrm{~A}$ and on $T=1,938^{\circ} \mathrm{K}$ at 0.5 ampere and $150 \mu$. This is probably the best of the three values of $T$, while the value derived from the slope is the least accurate. The large discrepancy for the two values given for a 2 -ampere discharge, at $33 \mu$, indicates that $T$ decreases with $n$ at this pressure, and it is obvious from figure 3 that it does this at $16 \mu$. 
The fact that the curves for the $\mathrm{S}$ and $\mathrm{F}$ series in figure 1 are parallel to the $\mathrm{D}$ series, indicates that all the series $\mathrm{S}, \mathrm{P}, \mathrm{D}$, and $\mathrm{F}$ have the same reversal temperature for values of $n$ above 5 . For equal values of $n$ the populations are assumed to be in the ratio 1:3:5:7. Numerical values are given by equation 2 with values of $T$ from table 3 . $N_{n}$ approaches a constant value as $n$ becomes large. This limiting value for $\mathrm{S}$ terms ranges from $2.2 \times 10^{5}$ at 0.5 ampere to $1.6 \times 10^{7}$ at 4 amperes for $150 \mu$, and it does not change much with pressure.

\section{TRANSITION PROBABILITIES}

The absolute value of $A_{n}$ can be computed from the values of $J$ and $N_{n}$. The $J$ value taken as standard for the last column of table 3 , viz, the intensity of $5635 \mathrm{~A}$ at 0.5 ampere and $150 \mu$ will be used. The numerical value of $\log J$ is 1.17 , in ergs per cubic centimeter. For comparison with hydrogen the intensity of the complete doublet is convenient and $\log J=1.35$. $\log N_{n}$ for the two $D$ states of weight 5 , at $1,938^{\circ} \mathrm{K}$, is 6.63 .

$$
A_{n}=J /\left(h_{\nu} \cdot N_{n}\right)=1.5 \times 10^{6} \text { per sec for } n=7.52
$$

For the $\mathrm{D}$ series from $n=4.52$ to higher numbers,

$$
A_{n}=\frac{6.4 \times 10^{8}}{n^{3}} \frac{\lambda_{n}}{5635} .
$$

The value for $5635 \mathrm{~A}$ for which $n=7.52$ can be compared with the hydrogen value for the transition $8_{2}$ to $2_{1}$ at $3889 \mathrm{~A}$. As $A_{n}$ is proportional to $\nu^{3}$ the cesium value should be multiplied by $(5635 / 3889)^{3}=$ 3.02 for the comparison. The hydrogen value ${ }^{7}$ is $2.1 \times 10^{6}$ and the cesium value reduced to the same wave length is $4.5 \times 10^{6}$.

In figure 1 the vertical distance between curves gives the log of the ratio of $J / \nu^{4}$ at equal values of $n$ or between terms of equal energy. The ratio for the red components of the $\mathrm{F}$ and $\mathrm{D}$ series is 5 , and the complete doublets will be in the ratio $5.6: 1$. The populations at equal $n$ values are in the ratio of $7: 5$, and the ratio of $A / \nu^{3}$ is $5 \times 5.6 / 7=$ 4.0. The hydrogen ratio of $A / \nu^{3}$ for the transitions $8_{3}$ to $3_{2}$ and $8_{2}$ to $2_{1}$ is 5.3 .

For the D and S series the ratio of $J / \nu^{4}$ is 10.5 , and as the populations are in the ratio $5: 1$, the values of $A / \nu^{3}$ are in the ratio $2.1: 1$. The hydrogen ratio is $16.7: 1$. While the transition probabilities in the $\mathrm{D}$ and $\mathrm{F}$ series are of the same magnitude as the theoretical hydrogen values, the cesium value for the $\mathrm{S}$ series is much higher.

Van der Held and Heierman ${ }^{8}$ obtained values for transition probabilities in potassium from measurements of the intensity of emission by a flame of known temperature containing potassium at a measured concentration. The value for $A_{n}$ for the $\mathrm{D}$ doublet 4966 and 4952 , $n=7.75$ is $0.38 \times 10^{6}$ as compared with $1.5 \times 10^{6}$ found in cesium for $n=7.52$. The value for the $\mathrm{S}$ doublet $4956 \mathrm{~A}$ and $4941 \mathrm{~A}$ is $1.01 \times 10^{6}$, while the corresponding value here is $0.71 \times 10^{6}$. The intensity variation in the potassium diffuse series is extremely abnormal for small values of $n$.

Ornstein and Key ${ }^{9}$ have published relative $A$ values for cesium based on measurements of the emission of a carbon arc containing

7 Handbuchder Physik 24, p. 444.

8 Van der Held and Heierman, Physica 3, 31 (1936).

$\checkmark$ Ornstein and Key, Physica 1, 945 (1934). 
cesium chloride, and on flame spectra measured by Miss Bleeker. ${ }^{10}$ The ratio of the $\mathrm{S}$ and $\mathrm{D}$ series is about that found here, but the variation of $A$ with $n$ is much faster than $1 / n^{3}$. Miss Bleeker has, however, concluded from her measurements that the population of $\mathrm{D}$ and $\mathrm{S}$ states did not have a temperature distribution. Certainly the intensity in a series drops more rapidly with increasing $n$ in a flame at $2,700^{\circ} \mathrm{K}$ than it does in the discharge at an effective temperature of $2,300^{\circ} \mathrm{K}$, and the results scarcely disprove the general rule that $A$ varies as $1 / n^{3}$ for large values of $n$.

\section{LINE SPECTRA AND CONTINUOUS SPECTRA}

In figure 4 it is seen that the intensity of diffuse series lines remains proportional to the continuous spectrum for higher currents and pressures, and from other data it is known that this is also true for the $\mathrm{S}$ and $\mathrm{F}$ series. With decreasing pressure the line intensity rises above the continuous spectrum. This indicates that at high pressures there is an equilibrium between the number of ionized states and the excited states which is maintained by recombination and ionization of excited states. At low pressures direct excitation by electron impact becomes predominant in maintaining the excitation. One important factor in this pressure change is known from studies of photoionization by line absorption. ${ }^{11}$ Radiation from excited states is quenched by atomic collision and for states of $n$. greater than 4.3 the quenching results in ionization in 15 to 100 percent of the collisions. At $40 \mu$ about 0.9 of the radiation is quenched. This increase of the ionization at the expense of the excited states will, with increasing pressure, bring the number of excited states into equilibrium with the number of ions. It is seen from the experimental results that a temperature distribution of the population of excited states is found in the range where ionized and excited states are in equilibrium and it is evidently not generally true at lower pressures.

It is shown in this work that the transition probabilities in the $\mathrm{D}$ and $\mathrm{F}$ series have approximately hydrogen values as measured at pressures from 70 to $350 \mu$ and in this range the continuous and line intensities are proportional. Published measurements of continuous intensity combined with measurements of the number of electrons per cubic centimeter ${ }^{12}$ have given values for the collision area $q$ for recombination into the $6 \mathrm{P}$ state. The results are not consistent with the line-transition probabilities. At pressures below $5 \mu, q$ has a value comparable to the theoretical hydrogen value and consistent with the $A$ values found for the cesium lines at high pressure, but the recombination measurements gave values of $q$ that increased rapidly with pressure to values far in excess of the hydrogen value in the pressure range where line-transition probabilities could be measured. The author now suspects that electron-concentration measurements made by the usual probe methods are systematically too low at high pressure and that $q$ is actually a constant. $q$ is a transition probability and the theoretical difficulties in understanding how it could change with pressure led to this attempt to evaluate line-transition probabilities by a method independent of probe measurements.

Washington, April 24, 1936.

10 Bleeker, Z. phys. Chem. 120, 63 (1926).

11 Mohler and Boeckner, BS J. Research 5, 51 (1930) RP 186.

12 Mohler, Recombination radiation in the cesium positive column, BS J. Research 10, 771 (1933) RP565. 